\title{
Active management of third stage of labor: Assessment of standard of care in one of the training institute
}

\author{
Meera Upadhyay, Gaurav Sharma, Dinesh Chataut \\ Consultant OBGYN \\ Lumbini Zonal Hospital, Butwal, Nepal.
}

\begin{abstract}
Objective: To find out the quality of service (standard steps of the procedures) incorporated in the active management of the third stage of labor (AMTSL) in Maternity Hospital, Thapathali, Kathmandu, Nepal.

Methods: Prospective (single blind) study, where the (uninformed) service providers had no knowledge that they were being assessed of their performances regarding the proper conduct of the AMTSL, in randomly selected 325 laboring women during all shifts of the day by 4 interns who carefully documented the findings in pre prepared standard 3 month duration reference during in the year 2061/2062 (2004/2005) in Maternity Hospital Thapathali, Kathmandu, Nepal.

Results: (Pre) loading of oxytocin was correctly done immediately when the women were seen to bear down at second stage of labor. In $99.5 \%$ of cases (irrespective of duty shift). In $5.8 \%$ of cases, the oxytocin doses were different from standard 10 units. The possibility of second twin was not ruled out in $81.9 \%$ before the administration of 10 units of IM oxytocin. Moreover, the study that controlled cord traction was revealed or showed applied in 50\% of the cases without confirming uterine contraction while counter traction to the uterus was given in most of the cases (98.5\%) along with effective examination of the placenta for its completeness in $98.2 \%$ cases.

Conclusion:This study shows that improvement in the standard AMTSL practices is still needed in the training providing institution, which demand ensuring the absence of twin tactfully before giving IM oxytocin within 2 minutes invariably after the delivery of the babies and also in giving slight traction on to the umbilical cord gently during uterine contraction along with counter traction to the uterus.
\end{abstract}

Key words: Active management of third stage of labor AMTSL, oxytocin, uterine massage.

\section{Introduction}

Active management of the third stage of labour (AMTSL) precisely includes administration of 10 units of intramuscular injection (IM) of oxytocin immediately within $1 \mathrm{~min}$ (some centers $2 \mathrm{~min}$ ) of the delivery of baby after ruling out the possibility of second baby [Oxytocin is being preferred to ergometrine because of its lesser side effects and (that) it can be given to any women with out intensive monitoring]. Then employing controlled cord traction when gentle traction is exerted into the umbilical cord during uterine contraction, simultaneously giving counter traction on the uterus which is slightly elevated above the pubis directed towards the umbilicus to avoid acute uterine inversion. Careful inspection of the placenta is done for its completeness once it is delivered, followed by uterine massage. ${ }^{1}$
AMTSL was launched in September 2002 by USAID and later co- supported by World Bank, UNFPA and the Swiss Cooperation to reduce postpartum hemorrhage and in July 29, 2004, Prevention of Postpartum Hemorrhage Initiative POPPHI project was awarded to a partnership of PATH, RTI International, EngenderHealth, the International Confederation of Midwives (ICM) and the International Federation of Gynecology and Obstetrics (FIGO) with special interest in reducing obstetric deaths. In one of the centre where AMSTL is practiced there were lesser number of post partum hemorrhages $0.4 \%$ for 2027 vaginal deliveries and a single maternal death $(0.05 \%)$ (April-Sept 2004) against $4.6 \%$ of post partum hemorrhages for 926 deliveries and 3 maternal deaths due to a post partum hemorrhage $0.3 \%$ (Saving Women's Lives One Birth at a Time, USAID: Benin).

\section{Correspondence}

Dr Meera Thapa Upadhyay, Consultant Gynecologist,

Lumbini Zonal Hospital, Butwal, email: ragat@wlink.com 
This study was carried out to verify the systematic application of AMTSL whether all the steps of AMTSL are being effectively covered during the conduction of third stage of labor and more emphasis could be laid on strengthening of the weakness for better outcome.

\section{Methods}

This is the prospective study where the conduct of third stage of labor by care providers who had no knowledge that they are being assessed (single blind) during all shifts of the day were observed by interns who documented each procedural steps in accordance to standard reference in randomly selected 325 laboring women were during the study period: BS 2061/2062 (2004/2005) in Prasutigirha, Maternity Hospital, Thapathali, one of the pioneer training centers in reproductive health was carried out.

Standard format for the steps of AMTSL (at vaginal delivery) with specified areas to be ticked was developed and following steps were tested.

1. $1^{\text {st }}$ step was the preparation of 10 units of Oxytocin as soon as the women have started bearing down in second stage of labor

2. How long after the delivery was oxytocin given

3. Whether the presence of twin was excluded before the administration of oxytocin or not.

4. Whether the controlled cord traction was undertaken during the uterine contraction or not.

5. The last steps was the inspection of the placenta for completeness.

\section{Results}

Health care providers attending 325 laboring women during the delivery were secretly observed (by Interns) to verify whether the active management of third stage of labor AMTSL was being systematically followed. Accordingly, the information were appropriately recorded (ticked) in the format and analyzed to find out whether there was any deviation from the standard way or not.

It was observed that the preparation of IM oxytocin (preloading of the medication) was done carefully in 99 $\%$ of the cases and had been effectively carried out even during the night shifts Table (1). But the doses incorporated were not the same in all the cases and very less $(5.8 \%)$ were presumed to receive oxytocin in doses other than the set in criteria. The oxytocin doses differed owing either to PPH or whenever the women were already receiving oxytocin drip for induction / augmentation of labour.

It was observed that exclusion of twin before giving IM oxytocin, an important step to avoid the fetal distress of the unborn twin or the entrapment of second twin was not carried in $260(80 \%)$ cases.

While the time interval between the delivery and injection of oxytocin was found to be within $2 \mathrm{~min}$ in 70 $\%$ (227.5) while the rest of the cases received oxytocin between 3- 9 min.

The study also critically evaluated missing one of the most important steps of exerting slight traction to the placental cord during uterine contraction at CCT which was carried out only in $50 \%$ of cases (Table 3). Whereas counter traction to the uterus was given in $98.5 \%$ of cases. In $98.2 \%$ of cases the placenta had been checked for completeness after delivery and also perineum and lower vagina was inspected in $97.8 \%$ of cases before shifting the patient.

\section{Discussion}

The active management of third stage of labor is one of the smallest interventions which Hinchingbrooke randomized control trial has significantly proven to lower the rate of PPH to $6.8 \%$ from that of expectant management $(16.5 \%)^{2}$. With the annual delivery rate of about 16000 (unpublished report) in this hospital, adaptation of the AMTSL is envisioned to reduce PPH. However this study was not on PPH. This is very important in Nepalese context where $80 \%$ of deliveries are still taking place at home even today, as described in progress report of Millennium Development Goal, $2005^{3}$ and adaptation of AMTSL, could mean a reduction in the rate of $\mathrm{PPH}$ arising from the faulty technique of placental delivery. Simply massaging the uterus through the abdomen as included in AMTSL until the uterus contracts/retracts can sufficiently prevent PPH which any skill birth attendant can be taught to practice without much of problem. Aware of the fact, that as low as $10-11 \%$ of births are being attended by skilled birth attendants (SBAs) or trained birth attendants (TBA) in Nepal, AMTSL being incorporated even in this small number of deliveries would help to reduce $\mathrm{PPH}^{4}$

The pros and cons derived from this urban based study finding inspires trainers to emphasize and highlight the right way to exclude the twin pregnancy before giving oxytocinon or perform CCT only when the uterus contracts and to massage the uterus when it appears to be flaccid.

The present study would have been definitely well appreciated if it had supplied information regarding PPH or retained placenta during the observation period. Especially in those cases who did not receive oxytocin within 2 min and $50 \%$ of the women who did not have gentle traction on the placental cord during uterine contraction and when $80 \%$ of twins were not excluded before giving oxytocinon. 
Table1. Oxytocin preparation /preloading

\begin{tabular}{lccc}
\hline Oxytocin preloading and administration & Evening & Morning & Night \\
Oxytocin preparation 323 (99.3\%) & 139 & 128 & 56 \\
Twin excluded 62 (19.1\%) & 22 & 29 & 11 \\
\hline
\end{tabular}

Table 2. Use of oxytocin

\begin{tabular}{lcc}
\hline Oxytocin doses and time of administration & Number & Percentage \\
Oxytocin dose (10unit) & 306 & $(94.2)$ \\
Administration carried out within 2 minutes & 227.5 & $(70 \%)$ \\
\hline
\end{tabular}

Table 3. Technique of placental delivery

\begin{tabular}{lcc}
\hline Techniques & Number & Percentage \\
Cord traction after assuring uterine contraction & 165 & $(50.8$ \\
Counter traction given & 320 & $(98.5)$ \\
Inspection of placenta for completeness & 319 & $(98.2)$ \\
\hline
\end{tabular}

General belief that the negligence is apt to occur at night shifts has been proven wrong in this study as the task was properly carried out during this phase.

However one of the pitfalls of this study is that, it does not denote who were the performers and how many times the same performers /conductors were taking the delivery as this could affect the report. Generally the normal deliveries in this hospital are conducted by ANM, staff nurses and nurses midwifes, junior interns (equivalent to medical clerk or final year MBBS), interns and medical officers. So there are wide variations. This study looked at the performances of the care providers but failed to address the consequences that were produced from the deviation from standard protocol described in AMSTL.

\section{Conclusion}

The criterion based audit to evaluate the quality of ongoing routine standard AMTSL practices has displayed a need to reemphasize some of the steps like exclusion of twin before administration of IM oxytocin that is supposed to be given within 2 minutes of delivery with the gentle umbilical cord traction being correctly applied during the uterine contraction at CCT especially in order to make improvements which are very vital to reduce complication in third stage of labor and ultimately the PPH, the feared killer that attributes to most of the maternal deaths in Nepal.

\section{Acknowledgement}

This study was completed with the financial support from Prasutigriha authority and research committee so we would like to thank all the staff and faculty especially Prof Sudha Sharma, for their help.

\section{References}

1. Mathai M and Sanghvi H, Ruidotti R. Normal labor and child birth. In: Integrated management of Pregnancy and Childbirth, a guide for midwives and doctors. Baltomore. JHPIEGO. 2000. C57-66.

2 RogersJ,Woodjet al. Active vs expectant management of third stage of labor; Hinchinabrooke randomized control trial. Lancet 1998; 351: 693-99

3 HMG Nepal, National Planning commission/UN country team of Nepal, Nepal Millennium Development Goals progress report 2005

4. FHD, DoHS, Ministry of Health. Maternal mortality and morbidity study.1998 